\title{
Various Modifications of Müller's Muscle-Conjunctival Resection for Ptosis Repair
}

\author{
Shu-Lang Liao" ${ }^{1}$, \\ Ann Yi-chiun Chuang ${ }^{2}$ \\ ${ }^{1}$ Department of Ophthalmology, National \\ Taiwan University Hospital, Taipei; \\ ${ }^{2}$ Department of Ophthalmology, Mackay \\ Memorial Hospital, Taipei, Taiwan
}

No potential conflict of interest relevant to this article was reported.

\begin{abstract}
Background The posterior approach for ptosis, including the Fasanella-Servat procedure and Müller's muscle-conjunctival resection (MMCR), has been proven effective for mild- to moderately affected patients whose levator muscle function remains relatively intact. Traditionally, MMCR was reserved for phenylephrine test-positive patients for whom various modifications and several nomograms have been developed. Methods We reviewed the literature for the Fasanella-Servat procedure and MMCR. The methods and results of the different modifications were analyzed and compared. Nomograms of these two procedures were summarized.

Results MMCR mainly involved resection of Müller's muscle and conjunctiva in the original design by Putterman and co-workers. The suggested resection ranged from 8 to 9 $\mathrm{mm}$ for the achievement of the maximal effect produced by topical epinephrine. Perry included tarsectomy in his procedure and developed a new nomogram that included a 1:1 ratio of the ptosis correction to the amount of tarsus resection. The effect of tarsectomy for the Fasanella-Servat procedure in terms of eyelid elevation was approximately one-half the value seen in phenylephrine test-negative patients. We've performed MMCR with tarsectomy regardless of the response to phenylephrine with similar results to Perry. Surgical indications and possible complications of MMCR with tarsectomy for Asian patients are discussed.

Conclusions MMCR is an effective and simple method for the correction of ptosis. With baseline $M M C R$, the additional tarsectomy has proven to be a powerful tool for ptosis correction irrespective of phenylephrine test results.
\end{abstract}

Keywords Blepharoplasty, Blepharoptosis

\section{INTRODUCTION}

Blepharoptosis, defined as an abnormal, low-lying upper eyelid margin, is considered a disfiguring, and sometimes vision-disabling, disease entity. Depending on the severity and cause of blepharop-

Received: Apr 1, 2015 Revised: Apr 14, 2015 Accepted: Apr 14, 2015 Correspondence: Ann Yi-chiun Chuang Department of Ophthalmology, Mackay Memorial Hospital, 92 Chung-Shan North Road, Taipei, Taiwan 10449. E-mail: eyeann@gmail.com

Copyright @ 2015 The Korean Society for Aesthetic Plastic Surgery.

This is an Open Access article distributed under the terms of the Creative Commons Attribution Non-Commercial License (http://creativecommons.org/licenses/by-nc/3.0/) which permits unrestricted non-commercial use, distribution, and reproduction in any medium, provided the original work is properly cited. www.e-aaps.org tosis, different surgical treatment modalities have evolved. Patients who demonstrate mild-to-moderate ptosis $(<3 \mathrm{~mm})$ with sufficient levator palpebrae supeioris function $(>8 \mathrm{~mm})$ are most likely to benefit from the posterior lamellar approach, mainly involving Müller's muscle-conjunctival resection (MMCR) with or without tarsectomy.

Evolution of the posterior lamellar approach

The posterior lamellar approach for the correction of blepharoptosis was first described in 1961 by Fasanella and Servat [1]. In this original method, two curved hemostats were used to incorporate 3 $\mathrm{mm}$ tarsus muscle, $3 \mathrm{~mm}$ Müller's muscle, and $6 \mathrm{~mm}$ overlying conjunctiva before a resection. The wound was closed with 4 to 5 interrupted or running 5-0 chromic sutures tied on the conjuncti- 
val side. In 1972, Putterman [2] modified the procedure using his newly designed serrated clamp with an inbuilt concave curvature in its central $14 \mathrm{~mm}$. The clamp had several advantages over the hemostat in terms of its better intraoperative handling and improved postoperative tarsus contour [2]. In 1975, Putterman and Urist [3] introduced the concept of MMCR, in which only the Müller's muscle and conjunctiva were resected, hence separating the two procedures in terms of the surgical technique, with the mechanisms and complications still shared between them.

\section{The falling popularity of the posterior approach}

Despite excellent surgical results, the development of the posterior approach was stalled by the awareness of the levator aponeurosis right around the same time. The proponents of levator aponeurosis surgery such as Jones et al. [4] and Anderson and Dixon [5] argued that since the defect of involutional ptosis is found to be in the aponeurosis instead of in the Müller's muscle or tarsus, it is improper to violate tissues not directly responsible for the disease. Arguments against posterior lamella surgery further included the plausible view of damaging the tear-producing structures (conjunctival goblet cells, meibomian glands, accessory lacrimal glands, and lacrimal gland ductules), causing dry eye and consequential corneal compromise. The "Age of aponeurotic awareness" in 1985 [6] directed the trend of ptosis surgery toward the anterior approach. Although some supporters of the posterior approach suggested that advancements in the levator aponeurosis was responsible for its success [1], most histopathologic studies argued against the incorporation of the levator aponeurosis in the resected specimen. Both Beard [7] and Buckman et al. [8] failed to identify the levator aponeurosis in resected specimens. Putterman [2] purposely designed his procedure to tease the conjunctiva and Müller's muscle from the overlying levator aponeurosis. Without the legitimacy of manipulating the levator aponeurosis, the posterior lamellar approach has thus fallen out of popularity since the 1980s.

\section{The reentry of the posterior approach}

The posterior approach later regained its popularity for several reasons. Although the levator aponeurosis can be directly visualized and corrected through an anterior approach, the surgical results are less predictable. Medial undercorrection due to the structurally weaker and less dynamic medial horn of the levator aponeurosis is a common complication [9]. A postoperative eyelid contour deformity is almost never encountered using the posterior approach. Compared to the posterior approach, higher surgical failure rates bias surgeons' preferences. McCulley et al. [10] reported that 8.7\% of levator advancement requires repeat surgery. Additionally, a 17.6\% repeat surgical rate for the external approach without blepharoplasty compared to $2.5 \%$ for the internal approach without blepharoplasty was reported by Ben Simon et al. [11]. Depending on the individual surgeons' surgical technique and grading criteria, the suc- cess rate of MMCR ranges from $85 \%$ to $98 \%[12,13]$. Time is another disadvantage of the external approach, which often requires tedious dissection and intraoperative adjustments that require a sitting position, whereas the posterior approach only requires preoperative planning and is relatively simple and anatomy-independent. MMCR takes less than 15 minutes per case, whereas a levator aponeurosis advancement through the anterior approach takes at least twice this amount of time. According to a survey conducted among members of the American Society of Ophthalmic Plastic and Reconstructive Surgeons (ASOPRS) in 2011 [14], 74\% of surgeons prefer the internal approach for ptosis correction.

\section{Modifications of MMCR}

Prior to Fasanella and Servat, the posterior approach for ptosis surgery involved an extensive dissection of the levator and the surrounding structures [15]. Fasanella and Servat [1] described a revolutionary approach of what was believed to be a combined levator-tarsusMMCR. The concept however was soon dismissed by Beard [7] who pointed out that it was actually a tarsus-MMCR by histopathological evidence. Putterman [2] deliberately avoided using a Desmarres retractor to evert the eyelid in order to apply traction only on the Müller's muscle-conjunctival complex. Putterman and Urist [3] published a sentinel modification of the resection confined to Müller's muscle and conjunctiva, attempting to avoid possible complications caused by tarsectomy (tarsal instability and keratitis sicca) in order to attribute the effectiveness of the procedure strictly to Müller's muscle. They also suggested that a resection of 8 to 8.25 mm of Müller's muscle duplicated the effect of epinephrine [16]. Improvements in surgical details such as the externalization of the tie [7], reintroduction of the Desmarres retractor [17], placement of traction (marking) sutures [18], and utilization of various sewing techniques/materials [19] further perfected the procedures. In 1991, Dresner [12] suggested a semi-linear nomogram based on the desired height of correction and response to $10 \%$ topical phenylephrine. If the response to phenylephrine was $2 \mathrm{~mm}$, for a desired elevation of $1 \mathrm{~mm}, 4 \mathrm{~mm}$ of Müller's muscle and conjunctiva was resected; $6 \mathrm{~mm}, 10 \mathrm{~mm}$, and $11 \mathrm{~mm}$ to $12 \mathrm{~mm}$ MMCRs, respectively, were performed if $2 \mathrm{~mm}, 3 \mathrm{~mm}$, and greater than or equal to $3 \mathrm{~mm}$ lid elevations were desired. Augmented or diminished resection by $1 \mathrm{~mm}$ to $2 \mathrm{~mm}$ are recommended for under- or over-response to phenylephrine, respectively. MMCR was limited to patients with minimal ptosis who had good phenylephrine response and good levator muscle function.

\section{MMCR with tarsectomy}

It was not until 2002 before tarsectomy was re-incorporated into the nomogram [20]. The modified algorithm by Perry et al. [20] was as follows: $9 \mathrm{~mm}$ of conjunctiva and Müller muscle $+\mathrm{x}$ mm of tarsus, in which $\mathrm{x}=$ distance of undercorrection after phenylephrine testing. Additional elevation was achieved by the resection of 
the tarsus at a 1:1 ratio. This algorithm granted surgeons a powerful tool, allowing for the correction of moderate to severe ptosis greater than $3 \mathrm{~mm}$ to $4 \mathrm{~mm}$ as long as the candidates possessed good levator muscle function and a fair phenylephrine response. For example, if $5 \mathrm{~mm}$ of the tarsus (the maximum allowable tarsus resection as suggested by Samimi et al. [21]) is resected, a possible $7 \mathrm{~mm}$ of ptosis correction could be achieved.

\section{Application of MMCR in phenylephrine test-negative patients}

Since one of the initial indications for MMCR is a positive test response to phenylephrine, few studies have investigated the effect of surgery on phenylephrine test-negative patients. However, it has been reported that the method is effective in those who test negative. Wee and Lee [22] showed that for patients who responded less than $2 \mathrm{~mm}$ to phenylephrine, the postoperative corrections were $1.19+0.78 \mathrm{~mm}$, approximately one-half that found in those with a phenylephrine response greater than $2 \mathrm{~mm}(2.41+0.84 \mathrm{~mm})$. Baldwin [23] performed open-sky MMCR on phenylephrine test-negative patients and was able to achieve $3.3 \mathrm{~mm}$ improvement of the upper eyelid margin reflex distance 1 (MRD1). He ensured optimal results by adjusting the amount of Müller's muscle resected (according to the height of the first suture) intraoperatively and altering the timing of suture removal postoperatively [23]. These results suggest that the Müller's muscle acts as the main transmitter of the levator muscle on the tarsal plate; shortening of it raises the upper eyelids regardless of whether it responds to phenylephrine [23,24].

\section{Differences between the Fasanella-Servat procedure and MMCR: Insight into the mechanisms of posterior lamellar surgery \\ Both procedures are effective for patients with mild-to-moderate ptosis and good levator muscle function.}

\section{Difference in surgical techniques}

The most obvious difference between the Fasanella-Servat procedure and MMCR is whether additional Müller's muscle above the tarsal border is resected. In the original report by Fasanella and Servat [1], it was claimed that the resected tissue specimen included $3 \mathrm{~mm}$ of tarsus, $3 \mathrm{~mm}$ of "levator- Müller," and $6 \mathrm{~mm}$ of conjunctiva. MMCR involved the active engagement of the lengthy Müller's muscle and conjunctiva superior to the tarsus. Fasanella and Servat [1] proposed the mechanism of levator aponeurosis resection and tarsus shortening. Putterman and Urist [3] believed that both procedures worked by Müller's muscle advancement and argued that tarsectomy played no role in the elevation of the eyelid. They based their argument on Obear's observation [25] that upper tarsal removal for a lower lid graft resulted in no lid level changes. With one exception [26], histopathologic studies have failed to detect the levator aponeurosis in specimens collected from both procedures [7]. Surprisingly, Buckman et al. [8] found that $87.5 \%$ of specimens collected from a Fasanella-Servat operation contained no or a minimal amount of smooth muscle. With no histopathological support to back up the role of one specific responsible tissue, Buckman et al. [8] concluded that the effectiveness of the Fasanella-Servat operation did not depend on Müllerectomy. Similarly, Zauberman et al. [27] found a lack of correlation between the amount of smooth muscle resected and the degree of improvement on ptosis in MMCR. How the posterior lamellar approach works for ptosis remains to be elucidated. Currently, it is possibly best explained by a combination of factors as Buckman et al. [8] suggested: (1) vertical posterior lamellar shortening; (2) secondary contractile cicatrization of the wound; and (3) plication or advancement of the Müller 's smooth muscle-levator aponeurosis complex on the tarsus.

\section{Difference in nomograms}

Traditionally, MMCR was reserved for patients with a positive response to phenylephrine and the Fasanella-Servat procedure was targeted toward those with suboptimal phenylephrine response. Without the removal of nearly $8 \mathrm{~mm}$ of the Müller's muscle-conjunctival flap, the nomogram appears to be quite different between the two procedures. The correlation between lid height correction to the amount of tarsectomy for MMCR is roughly 1 to 1 [20] while that for the Fasanella-Servat procedure was nearly two to one [21]. To date, no nomogram has been developed for MMCR with tarsectomy for phenylephrine test-negative patients.

\section{Liao's modification of MMCR}

We developed a unique procedure for combined MMCR and tarsectomy with no reference to the phenylephrine response [28]. Due to our previous surgical experience, MMCR alone without tarsectomy did not achieve satisfactory results in our Asian patients. We prefer combined MMCR with tarsectomy for posterior ptotic repairs. Prior to surgery, MRD1, lid fissures, and levator muscle function were recorded and photographs were taken. Slit lamp examination included ocular surface integrity, anterior segments, and possible lagophthalmos. A 2.5\% topical phenylephrine test was administered three times before the response was observed. In our experience, MMCR with tarsectomy may reduce an eyelid crease, which is quite an aesthetic concern for Asian populations. We chose patients with a high lid crease, minimal excess skin, and good levator muscle function ( $>8 \mathrm{~mm}$ ). For each case, $8 \mathrm{~mm}$ MMCR and 1 $\mathrm{mm}$ to $3 \mathrm{~mm}$ tarsectomy was performed. Specifically, if MRD1 was negative, $8 \mathrm{~mm}$ MMCR and $3 \mathrm{~mm}$ tarsectomy were the chosen amounts of resection. If MRD1 was $0 \mathrm{~mm}$ to $1 \mathrm{~mm}, 8 \mathrm{~mm}$ MMCR and $2 \mathrm{~mm}$ tarsectomy were done. When MRD1 was $2 \mathrm{~mm}$ to 3 $\mathrm{mm}, 8 \mathrm{~mm}$ MMCR and $1 \mathrm{~mm}$ tarsectomy were performed. For patients with fair levator muscle function $(5-7 \mathrm{~mm})$, an additional $1 \mathrm{~mm}$ of the tarsal plate was resected for better results. The results of the phenylephrine test alter the nomogram by $1 \mathrm{~mm}$ of tarsectomy. For example, if the phenylephrine test result was negative or 
suboptimal $(<2 \mathrm{~mm})$, we resected an additional $1 \mathrm{~mm}$ of the tarsal plate.

\section{Surgical procedure [28]}

Local anesthesia (2\% lidocaine with 1:150,000 epinephrine) was injected at the superior fornix and into the skin sites where the sutures were to be externalized. While the upper eyelid was everted over a Desmarres retractor, teeth forceps were used to tease the conjunctiva (and possibly some Müller's muscle) from the underlying tissues at one-half the desired resected length from the superior tarsal border. Three 4-0 silk traction sutures were placed centrally, medially, and temporally. With all three traction sutures pulled simultaneously toward the lower lid, a Putterman ptosis clamp was applied to the tarsal plate. The distances from the superior tarsal border to the central and lateral borders of the clamp ranged from $1 \mathrm{~mm}$ to $5 \mathrm{~mm}$ (Fig. 1). The medial distance should be about 0.5 $\mathrm{mm}$ greater to avoid postoperative medial ptosis. A 6-0 prolene suture was passed through the skin, exiting the medial end of the tarsus over the clamp. The suture is weaved 3 to 4 times through the tarsus $1 \mathrm{~mm}$ above the clamp toward the lateral end of the tarsal plate to the point at which the suture exited the skin and was then cut with enough length to tie. The same suture was re-introduced into the skin $1 \mathrm{~mm}$ from the previous exit site and was weaved medially through the tarsus in a baseball suture, exiting the skin at the end. The ends were tied together, and the clamped tarsal tissues were cut free along the border of the clamp (Fig. 2). Sutures were removed 14 days postoperatively. If an overcorrection was noted at the first visit (7 days), early removal of sutures with gentle massage may lower the lid height.

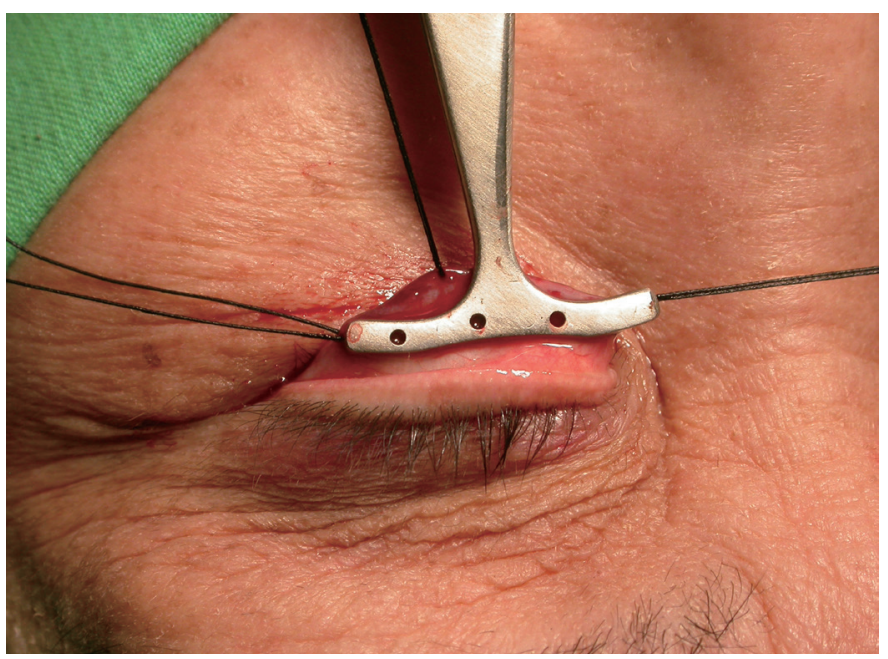

Fig. 1. With all three traction sutures pulled simultaneously toward the lower lid, a Putterman ptosis clamp was applied to the tarsal plate. The distances from the superior tarsal border to the central and the lateral borders of the clamp ranged from $1 \mathrm{~mm}$ to $5 \mathrm{~mm}$.

\section{Characteristics of Liao's method}

The surgical planning is relatively simple compared to previous nomograms. The resection of the tarsus in addition to MMCR accentuates the effects of tarsectomy to the same extent (i.e., 1:1 ratio), even when the correction due to MMCR was halved in phenylephrine test-negative patients. The results were predictable and satisfactory in terms of eyelid elevation and symmetry (Fig. 3-6). Although these results are only preliminary, we believe that with baseline MMCR, tarsectomy can be used as a predictable tool for lid height adjustment regardless of whether the phenylephrine test is positive.

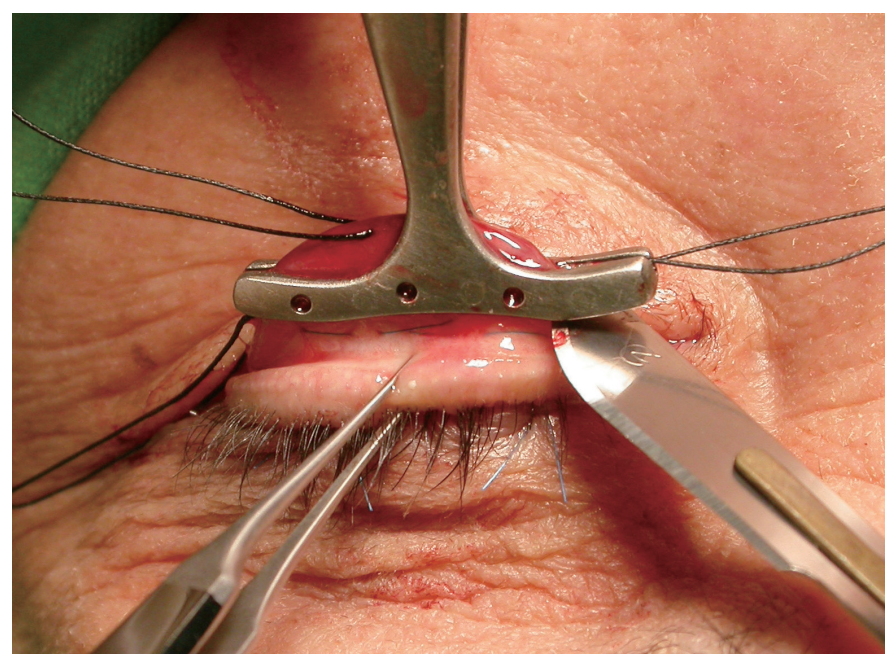

Fig. 2. After the ends were tied together, the clamped tarsal tissues were cut free along the border of the clamp.
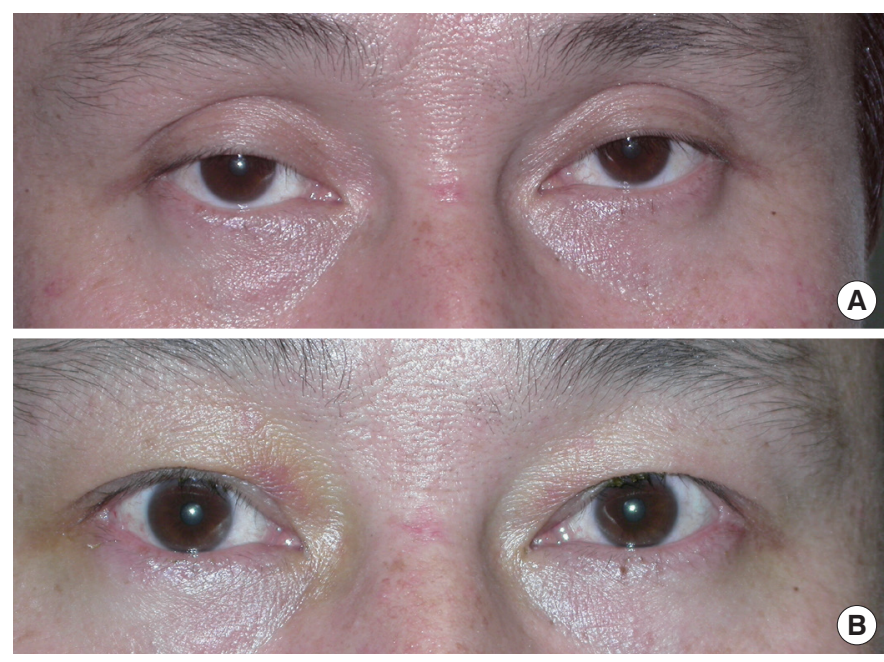

Fig. 3. (A) A 46-year-old male patient with high crease and good levator muscle function. (B) Bilateral ptosis improved after MMCR with $2 \mathrm{~mm}$ tarsectomy. 

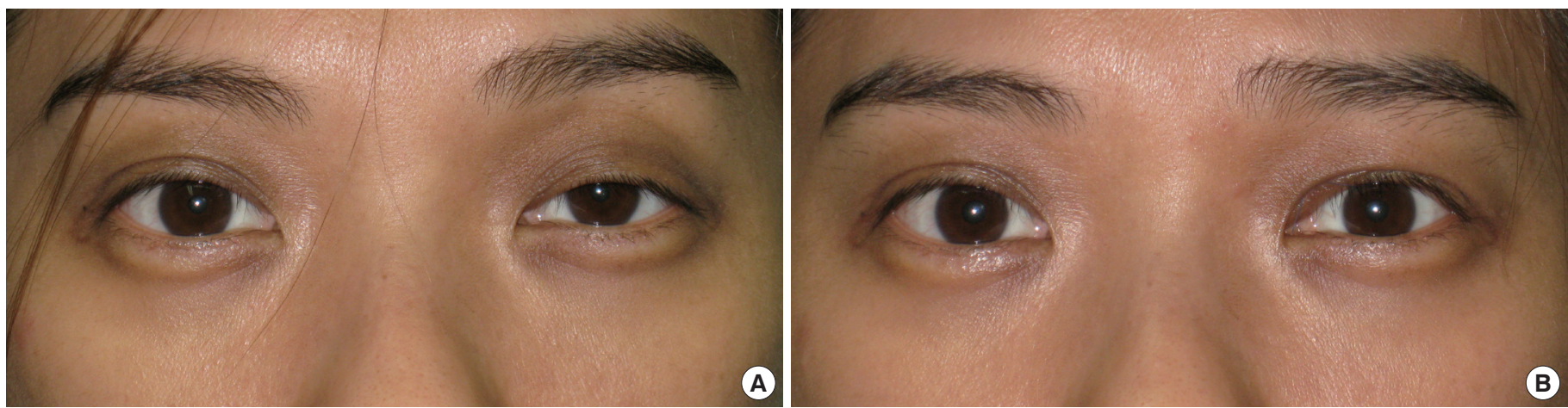

Fig. 4. (A) Thirty-year-old female patient with left eye ptosis, high crease and good levator muscle fuction. (B) Ptosis improved with satisfactory result 1 week after MMCR with $2 \mathrm{~mm}$ tarsectomy.
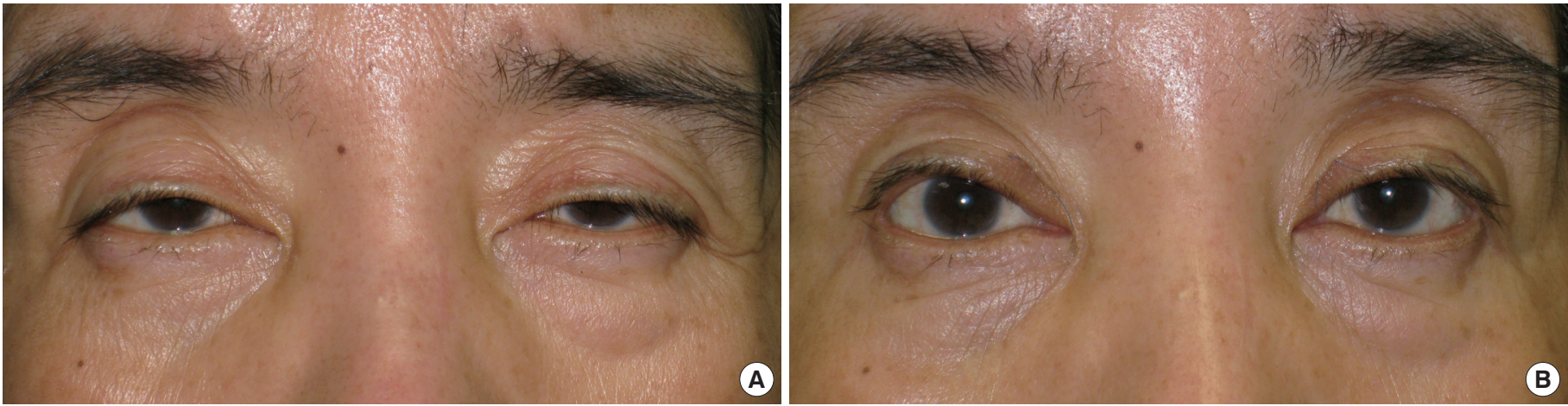

Fig. 5. (A) A 61-year-old female patient with bilateral severe ptosis and fair levator muscle fuction. (B) Bilateral ptosis improved with reasonable results 1 week after MMCR with $4 \mathrm{~mm}$ tarsectomy.
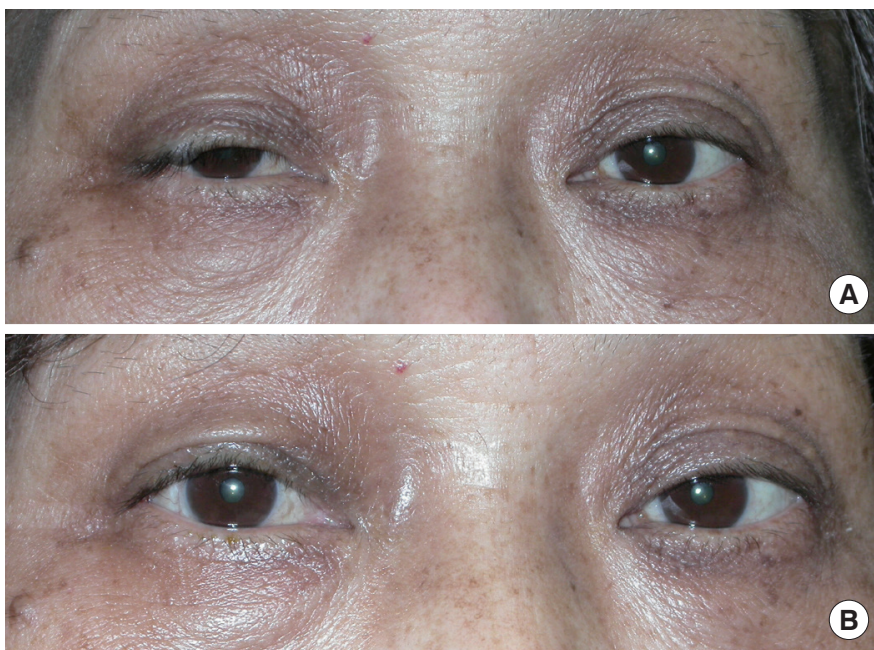

Fig. 6. (A) A 65-year-old female patient with right severe ptosis and fair levator muscle fuction. (B) Right eye ptosis improved with satisfactory result after MMCR with $4 \mathrm{~mm}$ tarsectomy.

\section{CONCLUSION}

MMCR with or without tarsectomy has become the treatment of
Table 1. Comparison of Müller's muscle-conjunctival resection with tarsectomy and levator muscle resection

\begin{tabular}{lcc}
\hline Methods & $\begin{array}{c}\text { MMCR with } \\
\text { tarsectomy }\end{array}$ & $\begin{array}{c}\text { Levator muscle } \\
\text { resection }\end{array}$ \\
\hline Operation time & 15 minutes & $30-45$ minutes \\
Recovery time & Short & Long \\
Visible scar & No & Yes \\
Predictability & Fair to good & Good \\
Difficulty & Simple & Difficult \\
Create crease & No & Yes \\
Satisfaction & Good & Fair
\end{tabular}

MMCR, Müller's muscle-conjunctival resection.

choice for patients with mild-to-moderate ptosis with a high lid crease and good levator muscle function. This posterior approach for ptosis has multiple advantages over the external approach (Table 1). The procedures are simple and case-independent, and the results are predictable with no compromise of the eyelid crease contour. Several nomograms have been developed for epinephrine test-positive patients as MMCR has traditionally been targeted for this patient group. Encouraging results have also been found in 
epinephrine test-negative patients when MMCR is combined with tarsectomy.

\section{PATIENT CONSENT}

Patients provided written consent for the use of their images.

\section{REFERENCES}

1. Fasanella RM, Servat J. Levator resection for minimal ptosis: another simplified operation. Arch Ophthalmol 1961;65:493-6.

2. Putterman AM. A clamp for strengthening Müller's muscle in the treatment of ptosis. Modification, theory, and clamp for the Fasanella-Servat ptosis operation. Arch Ophthalmol 1972;87:665-7.

3. Putterman AM, Urist MJ. Müller muscle-conjunctiva resection. Technique for treatment of blepharoptosis. Arch Ophthalmol 1975;93:61923.

4. Jones LT, Quickert MH, Wobig JL. The cure of ptosis by aponeurotic repair. Arch Ophthalmol 1975;93:629-34.

5. Anderson RL, Dixon RS. Aponeurotic ptosis surgery. Arch Ophthalmol 1979;97:1123-8.

6. Anderson RL. Age of aponeurotic awareness. Ophthal Plast Reconstr Surg 1985;1:77-9.

7. Beard C. Blepharoptosis repair by modified Fasanella-Servat operation. Am J Ophthalmol 1970;69:850-7.

8. Buckman G, Jakobiec FA, Hyde K, et al. Success of the Fasanella-Servat operation independent of Müller's smooth muscle excision. Ophthalmology 1989;96:413-8.

9. Kakizaki H, Zako M, Ide A, et al. Causes of undercorrection of medial palpebral fissures in blepharoptosis surgery. Ophthal Plast Reconstr Surg 2004;20:198-201.

10. McCulley TJ, Kersten RC, Kulwin DR, et al. Outcome and influencing factors of external levator palpebrae superioris aponeurosis advancement for blepharoptosis. Ophthal Plast Reconstr Surg 2003;19:388-93.

11. Ben Simon GJ, Lee S, Schwarcz RM, et al. External levator advancement vs Müller's muscle-conjunctival resection for correction of upper eyelid involutional ptosis. Am J Ophthalmol 2005;140:426-32.

12. Dresner SC. Further modifications of the Müller's muscle-conjunctival resection procedure for blepharoptosis. Ophthal Plast Reconstr Surg 1991;7:114-22.

13. Lake S, Mohammad-Ali FH, Khooshabeh R. Open sky Müller's mus- cle-conjunctiva resection for ptosis surgery. Eye (Lond) 2003;17:100812.

14. Aakalu VK, Setabutr P. Current ptosis management: a national survey of ASOPRS members. Ophthal Plast Reconstr Surg 2011;27:270-6.

15. Patel V, Malhotra R. Transconjunctival blepharoptosis surgery: a review of posterior approach ptosis surgery and posterior approach whiteline advancement. Open Ophthalmol J 2010;4:81-4.

16. Putterman AM, Fett DR. Müller's muscle in the treatment of upper eyelid retraction: a 12-year study. Ophthalmic Surg 1986;17:361-7.

17. Crawford JS. Repair of blepharoptosis with a modification of the Fasanella-Servat operation. Can J Ophthalmol 1973;8:19-23.

18. Weinstein GS, Buerger GF, Jr. Modification of the Müller's muscle-conjunctival resection operation for blepharoptosis. Am J Ophthalmol 1982;93:647-51.

19. Fox SA. A modified Fasanella-Servat procedure for ptosis. Arch Ophthalmol 1975;93:639-40.

20. Perry JD, Kadakia A, Foster JA. A new algorithm for ptosis repair using conjunctival Müllerectomy with or without tarsectomy. Ophthal Plast Reconstr Surg 2002;18:426-9.

21. Samimi DB, Erb MH, Lane CJ, et al. The modified fasanella-servat procedure: description and quantified analysis. Ophthal Plast Reconstr Surg 2013;29:30-4.

22. Wee SW, Lee JK. Clinical outcomes of conjunctiva-Müller muscle resection: association with phenylephrine test-negative blepharoptosis and dry eye syndrome. J Craniofac Surg 2014;25:898-901.

23. Baldwin HC, Bhagey J, Khooshabeh R. Open sky Müller muscle-conjunctival resection in phenylephrine test-negative blepharoptosis patients. Ophthal Plast Reconstr Surg 2005;21:276-80.

24. Bang YH, Park SH, Kim JH, et al. The role of Müller's muscle reconsidered. Plast Reconstr Surg 1998;101:1200-4.

25. Obear MF, Smith B. Tarsal grafting to elevate the lower lid margin. Am J Ophthalmol 1965;59:1088-90.

26. Morris CL, Morris WR, Fleming JC. A histological analysis of the Müllerectomy: redefining its mechanism in ptosis repair. Plast Reconstr Surg 2011;127:2333-41.

27. Zauberman NA, Koval T, Kinori M, et al. Müller's muscle-conjunctival resection for upper eyelid ptosis: correlation between amount of resected tissue and outcome. Br J Ophthalmol 2013;97:408-11.

28. Chung AYC, Liao SL. Periorbital rejuvenation surgery in the geriatric population. Int J Gerontol 2010;4:107-14. 